\title{
ARTICLE Expression of the oxygen-sensitive transcription factor subunit HIF-1 $\alpha$ in patients suffering from secondary Raynaud syndrome
}

Lukas Andreas Heger ${ }^{1}$, Mark Kerber ${ }^{1}$, Marcus Hortmann ${ }^{1}$, Samuel Robinson ${ }^{2}$, Maximilian Mauler ${ }^{1,3}$, Daniela Stallmann ${ }^{1}$, Daniel Duerschmied ${ }^{1}$, Christoph Bode ${ }^{1}$, Christoph Hehrlein ${ }^{1}$ and Ingo Ahrens ${ }^{1,4}$

Anti-ischemic therapy remains a challenge due to the complexity of hypoxia response pathways. Hypoxia-inducible factor (HIF)- 1 is a heterodimer transcription factor consisting of 2 subunits, HIF-1a and HIF-1 $\beta$. Hypoxia-dependent activation of HIF-1a regulates cellular $\mathrm{O}_{2}$ homeostasis. Raynaud syndrome (RS), as a comorbidity of the autoimmune disease systemic sclerosis (SS), is characterized by vasospasms that limit blood flow to the limbs, resulting in hypoxia. A single-center randomized study was conducted to compare prostaglandin E1 (PgE1) therapy with a treatment combining PgE1 and an endothelin-1 blocker, bosentan. A total of 30 patients suffering from SS with RS were enrolled. We examined the regulation of HIF-1a, its target heme oxygenase-1 (HMOX-1), and the serum levels of the HIF-1 a protein in a subset of patients as well as in ten healthy individuals. The expression of HIF-1a and HMOX-1 in monocytes was measured using absolute plasmid-based quantitative real-time PCR, whereas serum HIF-1a levels were measured with ELISA. Samples were taken at the time of randomization and after 24 weeks. We found that HIF-1a and HMOX-1 mRNA expression in monocytes and serum HIF-1a protein levels were significantly higher in the SS/RS patients compared to the healthy control group. Single-drug therapy significantly increased HIF-1a and HMOX-1 mRNA expression in monocytes and serum HIF-1a protein levels in the SS/RS patients compared to those at the time of randomization, whereas combining PgE1 with an endothelin-1 blocker prevented the further increases in HIF-1a and HMOX-1 expression. We propose HIF-1a and HMOX-1 as novel markers for anti-ischemic therapy in RS.

keywords: Raynaud syndrome; hypoxia; monocytes; HIF-1alpha; HMOX-1; anti-ischemic therapy

Acta Pharmacologica Sinica (2019) 40:500-506; https://doi.org/10.1038/s41401-018-0055-1

\section{INTRODUCTION}

Hypoxia-inducible factor (HIF)-1 is a heterodimer transcription factor that is upregulated under hypoxic conditions and plays a significant role in maintaining the body's $\mathrm{O}_{2}$ homeostasis [1]. HIF-1 regulates the expression of up to a hundred different genes involved in glucose metabolism, angiogenesis, vascular tone, and cell survival [2-5]. HIF-1 consists of a constitutively expressed beta subunit (HIF-1 $\beta$ ) and an oxygen-dependent alpha subunit (HIF-1a). Under normoxic conditions, HIF-1a is rapidly degraded by a proteasome, a process initiated by the von Hippel-Lindau tumor suppressor. However, under hypoxic conditions, degradation of HIF-1a is halted, and the subunit accumulates rapidly. The HIF-1a subunit is then able to translocate into the nucleus, where it dimerizes with HIF-1 $\beta$ and binds to short DNA sequences known as hypoxia response elements; this binding induces the transcription of certain genes such as heme oxygenase-1 (HMOX-1) and endothelin-1 (ET-1) [2]. Moreover, HIF-1 itself is subject to numerous regulatory mechanisms. For example, several studies have indicated that HIF-1 effector enzymes, such as HMOX-1 and ET-1, in turn stabilize HIF-1 expression and, when suppressed, lead to reductions in HIF-1 expression [6]. Its diversity of action and its complex regulation emphasize the importance of fully elucidating the role of HIF-1 in different pathological mechanisms $[7,8]$.

In patients suffering from autoimmune diseases, such as the connective tissue disease systemic sclerosis (SS), the extent of oxidative stress and hypoxia is associated with patient outcome and therapeutic success [9]. Consequently, HIF-1 has become of great interest in the current research in this field, particularly since it has been shown to be involved in persistent pathofibrogenesis and vascular remodeling in SS [10-12].

Increased oxygen consumption and tissue hypoxia not only contribute to the pathological hallmarks of SS-microangiopathy, autoimmunity, and tissue fibrosis-but also play significant roles in concomitant Raynaud syndrome (RS) $[10,11]$. RS is part of the classification criteria of the American College of Rheumatology (ACR) and the European League Against Rheumatism (EULAR) for SS and occurs in up to $98 \%$ of patients [12].

In affected patients, arterial vasospasm leads to intermittent periods of decreased blood flow in the extremities. RS episodes are often triggered by cold or emotional stress [13]. Affected

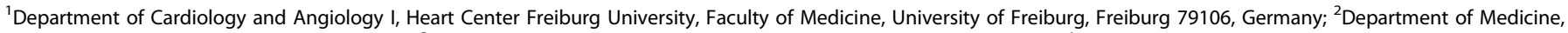

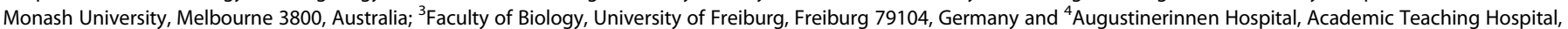
University of Cologne, Cologne 50678, Germany

Correspondence: Lukas Andreas Heger (Lukas.heger@universitaet-freiburg.de)

Received: 23 December 2017 Accepted: 30 May 2018

Published online: 10 July 2018 
Expression of HIF-1a in secondary Raynaud syndrome patients L. A. Heger et al.

extremities initially turn pale due to ischemia before turning blue from the stasis of deoxygenated blood. Although episodes are usually rapidly self-limiting, they can sometimes persist for hours. After the spasm resolves, the resulting reoxygenation turns the limbs red [14]. This cycle of transient vasospasm and resolution puts affected patients at risk of tissue damage through ischemia and reperfusion injury [15]. Frequent attacks of RS significantly limit patients' quality of life and can cause serious complications such as gangrene and the need for amputation [15].

In many patients, RS represents the initial manifestation of SS [11]; it has been postulated that RS contributes to the pathophysiological cascade leading to vascular damage and could be the initial trigger for the development of the disease $[15,16]$. Recent findings suggest that hypoxia itself, primarily through the activation of HIF-1 and the consequent stimulation of extracellular matrix molecules, including collagen and fibronectin, promotes structural changes in vessels [17].

Several compounds for pharmacotherapy in RS have been proposed and have been subject to limited clinical trials; however, at present, novel therapeutics lack sufficient evidence to support their use [18-20]. Refractory and/or severe symptoms are of particular interest for therapeutic research. Currently, patients with threatened or established digital ischemia are treated with intravenous prostanoids, such as prostaglandin E1 (PgE1) [21]. Furthermore, an orally administered inhibitor of the potent vasoconstrictor ET-1 is suggested to limit the frequency and severity of RS attacks [22].

As a potent regulator of immune and inflammatory reactions, monocytes often accumulate in hypoxic areas and, through the distribution of proangiogenic enzymes, participate in the adaptation to hypoxia [23]. A number of recent studies have demonstrated that monocytes adapt to hypoxia through upregulation of numerous transcription factors, including HIF-1 [23, 24]. In SS patients, monocytes have been found to contribute to oxidative stress via alteration in their cytokine language and the increased emission of reactive oxygen species (ROS) $[25,26]$.

This study aimed to (1) investigate HIF-1a expression in monocytes of SS/RS patients compared to those of healthy control individuals, (2) understand the effect of an established but off-label therapy on HIF-1a, and (3) correlate HIF-1a expression to serum levels of the HIF-1a protein and to the expression of the downstream effector protein HMOX-1 in monocytes.

\section{MATERIALS AND METHODS}

Subjects

In this prospective, single-center randomized controlled trial, we included 30 patients suffering from SS with RS (according to ACR guidelines) who were admitted to the Interdisciplinary Vascular Center of the University of Freiburg Heart Center from July 2011 to December 2015. The study was approved by the local ethics committee of the University of Freiburg (ethic number 58/11) and published as a clinical trial (NCT01378845).

Written consent was obtained from all patients according to the Declaration of Helsinki. After a screening during their first visit, patients were randomly selected and randomized to two treatment arms (1:1 ratio).

The first treatment group received a single PgE1 infusion therapy with alprostadil (Hexal AG, Industriestr. 25, 83607 Holzkirchen, Germany) at a dose of $60 \mu \mathrm{g}$ per day for 2 weeks. The second treatment group received a combination therapy composed of the aforementioned PgE1 regime and an additional oral treatment with the dual endothelin receptor blocker bosentan (Tracleer, Actelion Pharmaceuticals Dtl. GmbH, Konrad-GoldmannStraße 5B, 79100 Freiburg, Germany) in daily doses of $125 \mathrm{mg}$ for 4 weeks followed by daily doses of $250 \mathrm{mg}$ until the end of the study (24 weeks). Patients were excluded if they had received intravenous prostaglandins within the previous 4 weeks, had used

\begin{tabular}{|lll|}
\hline Table 1. & Adverse events & \\
\hline Patient ID & Adverse event & Medication \\
\hline 14 & Rhinitis & Combination therapy \\
16 & Dizziness & Combination therapy \\
& Skin rash & \\
& Hypertension & Combination therapy \\
17 & Leucopenia & Combination therapy \\
20 & Epigastralgia & ALT/AST elevation \\
22 & Rash & Single-drug therapy \\
\hline
\end{tabular}

phosphodiesterase inhibitors other than for intermittent treatment of male erectile dysfunction or had received inhaled or oral prostaglandins. Other exclusion criteria included a body weight $<$ $40 \mathrm{~kg}$, severe pulmonary arterial hypertension (WHO class III/IV), moderate to severe hepatic impairment or serum aminotransferase levels $>3$ times the upper limit of the normal range, and uncontrolled arterial hypertension. Patients were also excluded if they had received systemic antibiotics to treat infected digital ulcers prior to randomization. Contraceptive measures and monthly pregnancy testing were required before enrollment in the study. Safety was continually monitored to prevent adverse events such as headaches, edema, abnormal liver function, and anemia. In total, five patients were excluded from this clinical trial due to adverse events (Table 1).

\section{Biochemical measurements}

We collected blood samples from included patients at the time of enrollment and at the end of the study (after 24 weeks). Blood was also obtained from a control group of 10 healthy donors. Peripheral venous blood was collected and processed within $2 \mathrm{~h}$; serum was also collected and left to clot at room temperature for $30 \mathrm{~min}$ before being centrifuged for $10 \mathrm{~min}$ at $2000 \mathrm{rpm}$. Serum samples were aliquoted and stored at $-80^{\circ} \mathrm{C}$.

\section{Purification of monocytes}

Blood samples were layered in equal volumes onto Ficoll density centrifugation media (Biocoll Separating Solution; Biochrom; Leonorenstraße 2, 12247 Berlin, Germany) and centrifuged at $2000 \mathrm{rpm}$ for $20 \mathrm{~min}$. Thereafter, the interphase containing peripheral blood mononuclear cells (PBMC) was carefully aspirated and divided into 2 tubes. After thorough decontamination by repeated pelleting and resuspension, each sample was stained with Trypan blue (Thermo Fisher Scientific Inc. 15250-061) and the exact number of isolated mononuclear cells was determined in a Neubauer counting chamber (Celeromics Technologies, Av de la Plata, 113, 46006 Valencia, Spain). Monocytes were isolated from PBMCs through magnetic cell separation by depletion of nonmonocytes and were labeled using a cocktail of biotin-conjugated antibodies and Anti-Biotin MicroBeads (Monocyte Isolation Kit II, human; Miltenyi Biotec GmbH, 51427, Germany).

\section{Absolute quantification of copy number}

The enriched monocyte suspension was again pelleted, and Trizol reagent (Ambion Life Technologies, CA, USA) was used to extract RNA according to the manufacturer's instructions. After spectral photometric determination of RNA quality and quantity, we generated CDNA templates for further PCR amplification and downstream experiments (Maxima First Strand CDNA Synthesis Kit for RT-qPCR with dsDNase, Thermo Fisher Scientific, MA, USA). To create a reproducible and reliable standard curve for the following qPCR, we equipped a plasmid with our cloned target sequences using a pGEM-T Easy Vector System (Promega; Madison, WI, USA). The material containing the constructed plasmids was sent to 
Table 2. Patient characteristics

\begin{tabular}{|c|c|c|c|}
\hline & $\begin{array}{l}\text { Single-drug } \\
\text { therapy }\end{array}$ & $\begin{array}{l}\text { Combination } \\
\text { therapy }\end{array}$ & $P$-value \\
\hline Age & $53.87( \pm 14.23)$ & $54.73( \pm 12.69)$ & 0.8615 \\
\hline Women \% & $56 \%$ & $79 \%$ & $0.0005^{*}$ \\
\hline Weight $(\mathrm{kg})$ & $73.53( \pm 20)$ & $72.07( \pm 16.14)$ & 0.8267 \\
\hline Diffuse SS type \% & $56 \%$ & $53.3 \%$ & 0.6701 \\
\hline Ca blockers \% & $37 \%$ & $37 \%$ & 1 \\
\hline Nitroglycerin \% & $6.7 \%$ & $6.7 \%$ & 1 \\
\hline Phosphodiesterase \% & $6.7 \%$ & $6.7 \%$ & 1 \\
\hline Anticoagulants \% & $56.2 \%$ & $42 \%$ & $0.0047^{*}$ \\
\hline Immunomodulators \% & $56.2 \%$ & $42 \%$ & $0.0047^{*}$ \\
\hline $\begin{array}{l}\text { Digital ulcers at the time of } \\
\text { inclusion (previous } / 1 / \geq 2 \text { ) \% }\end{array}$ & $46 \% / 33 \% / 26 \%$ & $40 \% / 33 \% / 20 \%$ & 0.9623 \\
\hline $\begin{array}{l}\text { Cigarette abuse (never/ } \\
\text { previous/active) }\end{array}$ & $60 \% / 20 \% / 26 \%$ & $40 \% / 20 \% / 33 \%$ & 0.7481 \\
\hline
\end{tabular}

Eurofins MWG Operon (85560 Ebersberg, Germany) for sequencing. Standards were obtained by serial dilution of the dissolved plasmids.

Using the aforementioned plasmid-based standard curve, we quantified the absolute copy number of our target gene with the double-stranded DNA-binding dye SYBR Green (Roche, Mannheim, Germany) using RT-qPCR. Runs were analyzed by LightCycler 480 Software, Version 1.5. All results were measured in duplicate, and the mean of each set of duplicates was used in further analysis.

GAPDH was used as an endogenous control gene to normalize all results. All primers were designed using Primer-Blast and were acquired from Eurofins MWG Operon in 85560 Ebersberg Germany. The sequences of the primers were as follows (forward, reverse): TGAAGACATCGCGGGGACCG, TTCGCCGAGATCTGGCTG CATC for HIF-1a; AGGTGACCCGAGACGGCTTCA, GGGCGAAGAC TGGGCTCTCCT for HMOX-1; and GCTTCCTGCGAACACACAAG, TCCATCTGCTGGTCAGCTTC for HIF-2a.

Serum HIF-1a protein level

We measured the HIF-1a protein level in blood samples using a human HIF-1a ELISA kit (Abcam plc, Taipei City, Taiwan).

Statistical analysis

The primary objective of this study was to analyze the HIF-1a and HMOX-1 mRNA expression in monocytes and the HIF-1a protein levels in the serum of SS/RS patients and to compare the expression and protein levels of patients to those in a healthy control group. A further aim was to investigate HIF-1a and HMOX1 mRNA expression in the SS/RS group after two different therapy regimes. On this basis, a total of 30 patients and 10 healthy controls were enrolled in this study for analysis, and the patients were randomized to either treatment arms.

Values were tested for a Gaussian distribution using the D'Agostino-Pearson omnibus normality test with a $95 \%$ confidence level. Continuous variables are presented as medians \pm lower and upper quartiles if they followed a non-Gaussian distribution and as means \pm SEM if they followed a Gaussian distribution. Categorical patient characteristics are presented as percentages. Non-normally distributed variables were tested using the Mann-Whitney U-test, and normally distributed values were tested using Student's $t$-test. Categorical differences between patient groups were compared using a Chi-square analysis.
Mixed ANOVA followed by post hoc Bonferroni testing was used to determine differences between the two paired patient groups.

A $P$-value of $\leq 0.05$ was considered statistically significant for all analyses. All analyses were performed using GraphPad Prism Version 6.0 (Prism 6 for Mac OS X; GraphPad Software, Inc., La Jolla, (A, USA).

\section{RESULTS}

\section{Patient characteristics}

The study population was composed of $66.7 \%$ women and $33.3 \%$ men. The average age across all patients was $54.30 \pm 13.25$ years. Table 2 illustrates the main baseline epidemiological and clinical data of the patients. The baseline characteristics of these two groups included age; weight; cigarette abuse; SS subtype, defined as either diffuse or limited disease; the number of digital ulcers at the time of inclusion; and current medications, including Ca-channel blockers, nitroglycerin, phosphodiesterase inhibitors, anticoagulants, and immunomodulators. There were significant differences in the gender composition and number of patients treated with immunomodulators and anticoagulants $(P=0.0005$, $P=0.0047, P=0.0047$ ) between the two therapy arms. There were no other significant differences in baseline clinical characteristics.

HIF-1a protein in serum and HIF-1a mRNA expression in monocytes of patients with RS and SS compared to a healthy control group

Patients with SS/RS at the time of inclusion $(n=30)$ demonstrated significantly higher serum levels of HIF-1a compared to the healthy control group $(n=10)$ (166.5 (IQR 120-259.8) pg/mL vs. 76 (IQR 47-110) $\mathrm{pg} / \mathrm{mL} ; P=0.0004$ ) (Fig. 1a).

RT-qPCR analysis demonstrated that monocyte expression of HIF-1a mRNA was significantly increased in patients with SS/RS compared to the healthy control group (1325 (IQR 430.6-2505) copies/ $\mu \mathrm{L}$ vs. 257.8 (IQR 132.3-858.6) copies/ $\mu \mathrm{L} ; \quad P=0.0047$ ) (Fig. 1b).

HIF-1a protein levels in patient serum and mRNA expression in monocytes of affected patients before and after the two therapy regimes

Serum levels of HIF-1a were significantly increased in patients treated with single-drug therapy for 24 weeks compared to the baseline levels at the time of inclusion (426 (IQR 243-703) pg/mL vs. 204 (IQR 129-325) pg/mL; $P=0.0002$ ). In patients treated with combination therapy, there was no significant difference between serum HIF-1a levels after 24 weeks of treatment and baseline levels (156 (IQR 97-241) pg/mL vs. 151 (IQR 117-220) pg/mL; $P>0.999)$. At the end of the study, serum HIF-1a levels were significantly higher in single-therapy patients compared to the levels in those who received combination therapy (426 (IQR 243-703) $\mathrm{pg} / \mathrm{mL}$ vs. 156 (IQR 97-241) $\mathrm{pg} / \mathrm{mL} ; P=0.0094)$.

RT-qPCR analysis demonstrated significantly increased HIF-1a mRNA expression in isolated monocytes from patients treated with single-drug therapy after 24 weeks compared to the expression at the time of inclusion (4111 (IQR 2320-6274) copies/ $\mu \mathrm{L}$ vs. 1040 (IQR 402.7-2914) copies per $\mu \mathrm{L} ; P<0.0001$ ). Patients treated with combination therapy demonstrated a trend toward lower expression of monocyte HIF-1a mRNA after 24 weeks of treatment compared to the expression at the time of inclusion, but the difference was not significant (733 (IQR 330-1313) copies/ $\mu \mathrm{L}$ vs. 1897 (IQR 445-2451) copies/ $\mu \mathrm{L} ; P=0.4292$ ). At the end of the study, patients who received single-drug therapy showed significantly increased HIF-1a mRNA expression compared to those who received combination therapy (4111 (IQR 2320-6274) copies/ $\mu \mathrm{L}$ vs. 733 (IQR 330-1313) copies/ $\mu \mathrm{L} ; P<0.0001$ ). There was no significant difference between the two treatment groups in the expression at the time of inclusion $(P>0.9999)$ (Fig. 2). 

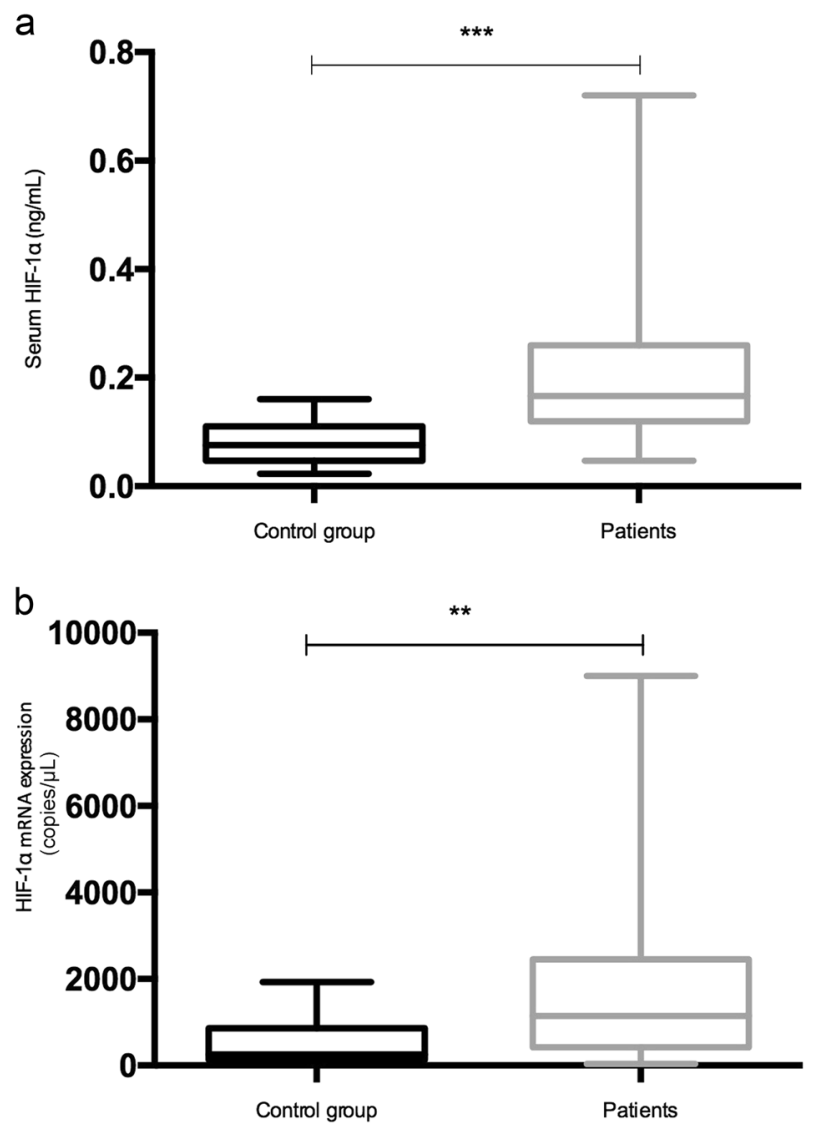

Fig. 1 HIF- $1 \alpha$ serum levels (a) and HIF- $1 \alpha$ mRNA expression levels in the monocytes (b) from the members of a healthy control group $(n=10)$ and patients with SS/RS $(n=30)$ at the time of inclusion. Data are presented as box plots with median and interquartile range. ${ }^{* *} P<0.01,{ }^{* * *} P<0.001$

HIF-1a target gene HMOX-1 expression in SS/RS patients Included patients with SS/RS demonstrated significantly increased expression of HMOX-1 mRNA in monocytes compared to the healthy control group (74.9 SD \pm 63.9 copies/ $\mu \mathrm{L}$ vs. $18.01 \mathrm{SD} \pm$ 14.11 copies $/ \mu \mathrm{L} ; P=0.0087)$. Patients treated with single-drug therapy showed significantly increased HMOX-1 mRNA expression after 24 weeks compared to expression at the time of inclusion (204 (IQR 84-270) copies/ $\mu \mathrm{L}$ vs. 49.9 (IQR 2.02-135) copies/ $\mu \mathrm{L} ; P=$ $0.0008)$. There was no significant difference in HMOX-1 mRNA expression in patients treated with combination therapy after 24 weeks compared to the expression at the time of inclusion (41.9 (IQR 10.9-132) copies/ $\mu \mathrm{L}$ vs. 68.1 (IQR 23.8-162) copies/ $\mu \mathrm{L}$ ). At the end of the study, patients treated with single-drug therapy demonstrated significantly higher expression levels of HMOX-1 mRNA compared to patients treated with combination therapy (204 (IQR 84-270) copies/ $\mu \mathrm{L}$ vs. 41.9 (IQR 10.9-132) copies/ $\mu \mathrm{L} ; P=$ 0.0014 ) (Fig. 3).

HIF-2a expression is less pronounced than HIF-1a expression but seems to follow similar expression patterns

Patients with SS/RS did not demonstrate significant differences in HIF-2a mRNA expression compared to the healthy control group

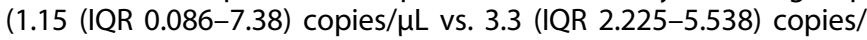
$\mu \mathrm{L})$. SS/RS patients treated with single-drug therapy demonstrated increased HIF-2a mRNA expression in isolated monocytes after 24 weeks of therapy compared to the levels at the time of inclusion (28 (IQR 13.5-56.4) copies/ $\mu \mathrm{L}$ vs. 1.40 (IQR 0.0446-5.538) copies/ $\mu \mathrm{L} ; P=0.0014)$. Patients treated with combination therapy
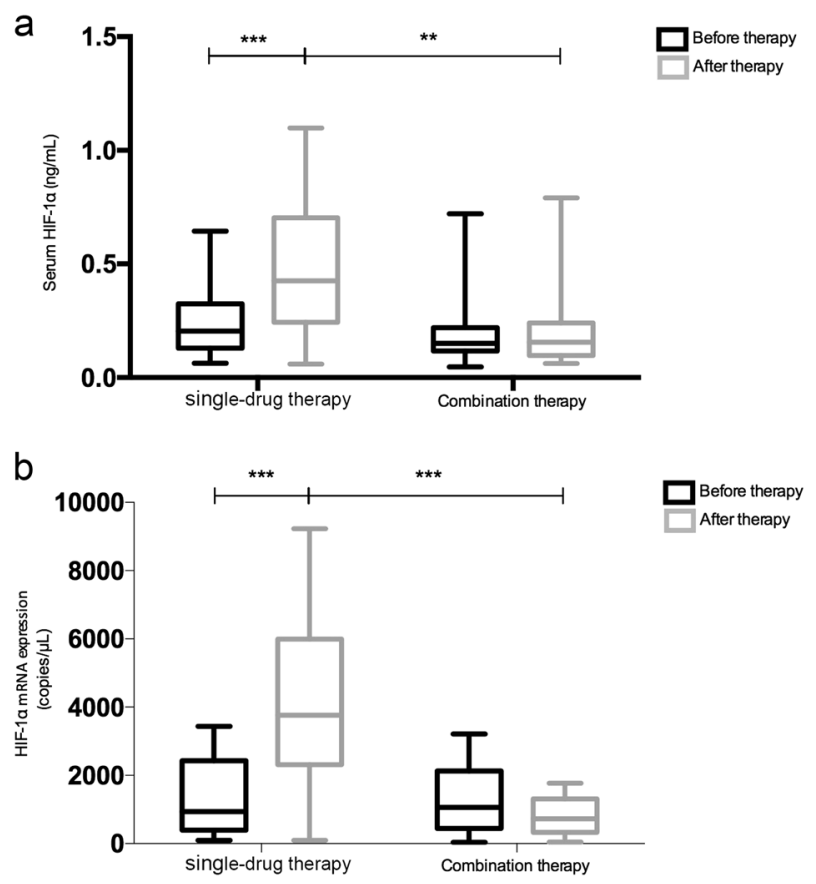

Fig. 2 HIF-1 $\alpha$ serum levels (a) and HIF- $1 \alpha$ mRNA expression in the monocytes (b) from included patients $(n=30)$ before and after a 24-week treatment period with either single-drug therapy or combination therapy. Data are presented as box plots with medians and interquartile ranges. ${ }^{* *} P<0.01,{ }^{* * *} P<0.001$

demonstrated no significant differences in HIF-2a mRNA expression after 24 weeks of therapy compared to the levels at the time of inclusion (4.89 (IQR 0.475-19.27) copies/ $\mu \mathrm{L}$ vs. 1.15 (IQR $0.1-7.35$ ) copies $/ \mu \mathrm{L} ; P=0.9999$ ) (Fig. 4).

At the end of the study, patients with SS/RS treated with singledrug therapy showed higher levels of HIF-2a mRNA expression compared to those treated with combination therapy (28 (IQR 13.5-56.4) copies/ $\mu$ L vs. 4.89 (IQR 0.475-19.27) copies/ $\mu \mathrm{L} ; P=$ 0.0019). There was no significant difference between the two treatment groups in the expression at the time of inclusion $(P>$ 0.9999).

\section{DISCUSSION}

In this study, we investigated, for the first time, the expression of HIF-1 a mRNA in the monocytes of patients suffering from SS with RS. Furthermore, we compared the effects of 2 RS treatment regimes on HIF-1 a mRNA expression in monocytes.

The main objective of this study was to investigate whether there is a distinct alteration in HIF-1a mRNA expression in the monocytes of affected patients compared to healthy individuals and whether currently established treatment regimes affect HIF1a mRNA expression.

We also investigated the concentrations of the HIF-1a protein in serum and the expression of the HIF-1 effector enzyme HMOX-1 in the monocytes of patients under the two different treatment regimes.

The alpha subunit of the heterodimer transcription factor HIF-1 (HIF-1a) belongs to the basic helix-loop-helix/Per-ARNT-Sim subfamily of eukaryotic transcription factors. In normoxia, HIF-1a is a target for rapid proteasome degradation initiated by the von Hippel-Lindau tumor suppressor. However, HIF-1a accumulates rapidly during hypoxic states. Upon accumulation, the HIF-1a subunit is able to translocate into the nucleus, where it dimerizes with its counterpart HIF-1 $\beta$ and binds to hypoxia response elements that are located within $\mathrm{O}_{2}$-regulating genes $[1,7,8,27]$. 

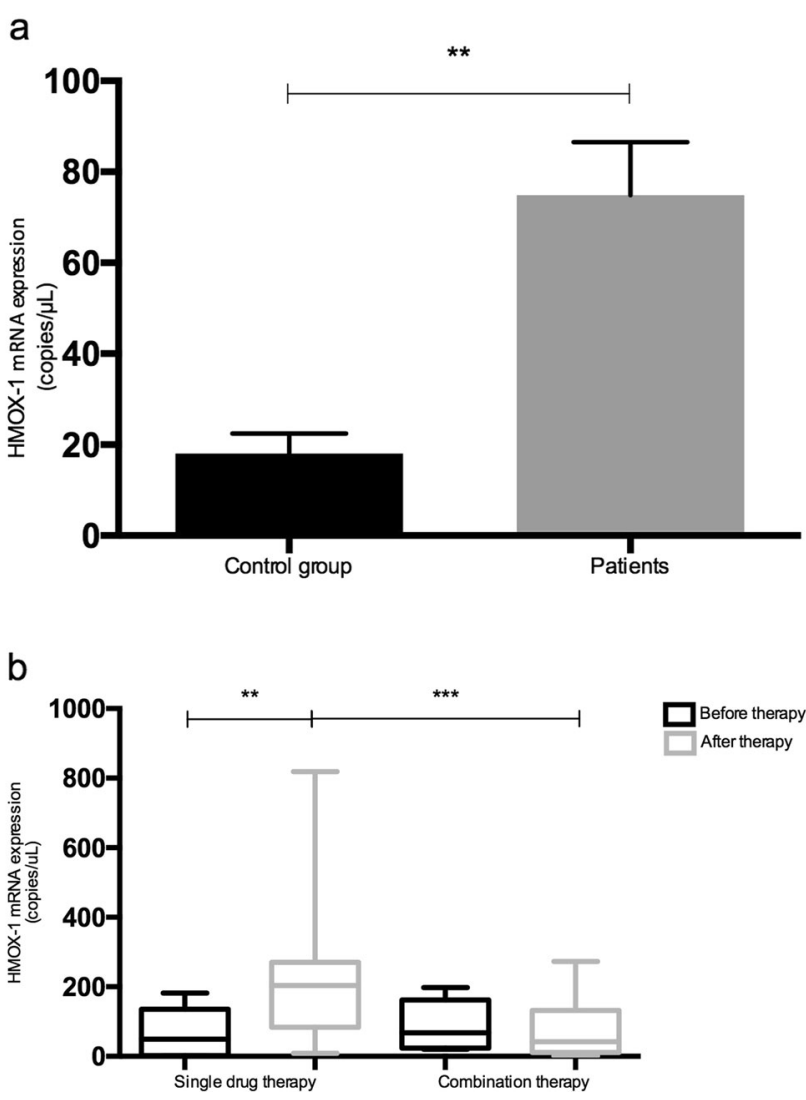

Fig. 3 a HMOX-1 mRNA expression in the monocytes of included patients $(n=30)$ compared to the expression in a healthy control group $(n=10)$. Data are presented as the means \pm SEM. b HMOX-1 mRNA expression in the monocytes of included patients before and after a 24-week treatment period with either single-drug therapy or combination therapy. Data are presented as box plots with medians and interquartile ranges. $(n=30)$. ${ }^{* *} P<0.01,{ }^{* * *} P<0.001$

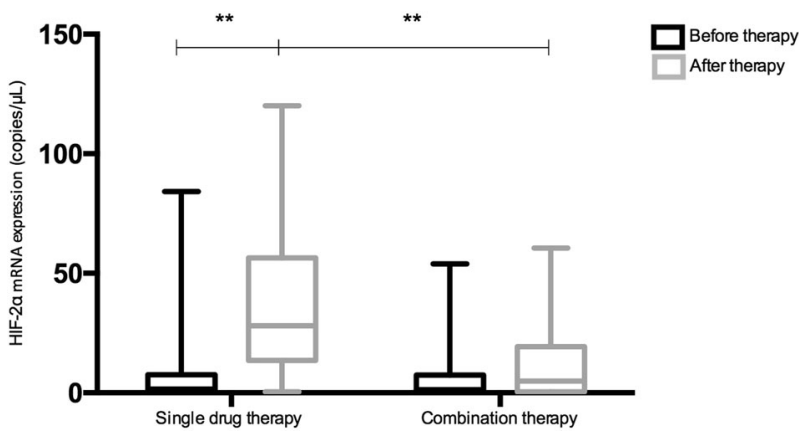

Fig. 4 HIF-2 $\alpha$ mRNA expression in the monocytes of included patients before and after a 24-week treatment period with either single-drug therapy or combination therapy. Data are presented as box plots with medians and interquartile ranges. $(n=30) .{ }^{* *} P<0.01$

Recent studies have indicated a central role of HIF-1 in influencing the adaptation and response of human monocytes to hypoxia $[24,28]$.

Despite its intracellular location, HIF-1a can be detected circulating in serum. Some evidence suggests that the serum levels of HIF-1a may be useful biomarkers for patient outcome, and high serum HIF-1a levels may be an independent risk factor in coronary artery calcification [29]. HIF-1 affects and induces the expression of numerous genes involved in maintaining homeostasis as oxygen concentrations change, including HMOX-1 [4]. Similar to HIF-1, HMOX-1 is thought to participate in the activation and distribution of mononuclear cells, thereby modulating the response to tissue hypoxia $[30,31]$.

The study of the influence and integration of the physiological response and adaptation to hypoxia is a highly interesting field of current medical research.

This study aimed to build on existing foundations and analyze the effects of two different treatment regimes on HIF-1a expression [32, 33].

Aside from its role in the body's response to hypoxia, several studies have demonstrated a potential role of HIF-1 in SS pathogenesis and susceptibility [17, 34]. Some studies have suggested that HIF plays a central role in tissue fibrosis, one of the pathophysiological hallmarks of SS [35].

In this study, we have described, for the first time (to the best of our knowledge), significantly increased levels of HIF-1a mRNA expression in the monocytes of patients suffering from SS with RS compared to the levels in healthy individuals. We have also demonstrated alterations in HIF-1a mRNA expression in the monocytes of patients treated with different therapy regimes. Moreover, we have analyzed HIF-1a protein expression in serum, which demonstrated patterns similar to those of monocyte mRNA expression. Specifically, HIF-1a protein levels were higher in patients with SS/RS than in healthy controls, and the levels were distinctly different between treatment regimes within the patient population. We believe that these results offer further insight into the mechanisms regulating HIF-1 expression, particularly with regard to the current idea that HIF-1 is mainly regulated by changes in protein levels [36].

A possible explanation for the elevated HIF-1a mRNA levels in the monocytes of patients suffering from SS/RS could be the repetitive cycles of hypoxia and reoxygenation that occur in Raynaud attacks [37]. Recent studies have indicated that free radicals (i.e., ROS) that are released during such attacks induce HIF1a expression [37, 38]. Studies have also suggested that one trigger of Raynaud attacks, exposure to cold weather, itself induces ROS production, fueling the HIF-1a induction partly through an ET-1-dependent pathway [39]. Furthermore, studies have shown that ET-1, a vasoactive protein that is known to be overexpressed in patients suffering from SS, stabilizes HIF-1a and is induced by HIF-1 [40, 41]. This suggests that HIF-1a may be involved in a self-perpetuating cycle via the induction of ET-1.

The aberrant HIF-1 physiology associated with RS suggests that pharmacological targeting of HIF-1a expression may have an impact on both the frequency and severity of Raynaud attacks. In several studies, treatment with an endothelin blocker has been associated with a decline in the occurrence of digital ulcers $[19,22]$. Moreover, the results of several studies support the use of combination treatment in affected patients. In particular, the use of vasodilating agents in combination with endothelin receptor antagonists has shown therapeutic benefit $[42,43]$.

In this study, HIF-1a expression proved to be a distinctive marker in differentiating between the two applied treatment regimes. At the end of the study (after 24 weeks), patients treated with single-drug therapy demonstrated significantly increased HIF-1a expression, as well as increased HIF-1a serum levels, compared to baseline levels, while patients treated with combination therapy demonstrated nonsignificant differences in HIF-1 a expression with a trend toward lower expression. We argue that the lower expression of HIF-1a at the end of therapy suggests the superiority of combination therapy. Despite the positive effects of HIF-1, it seems to be a marker of hypoxia and may contribute to the progression of $\mathrm{RS}[7,8,35]$. The higher expression of HIF-1a in single-therapy patients is likely due to disease progression. However, the short half-life of the single-drug therapy combined with the long interval in between testing is a 
possible confounding factor [44]. In contrast, the combination regime included the same single-therapy alprostadil regime combined with additional continuous daily treatment with an endothelin receptor blocker.

HIF-1a protein expression in serum has been shown to be an independent marker for patient outcome in acute ischemic stroke, and studies have demonstrated that high serum HIF-1a levels are likely a risk factor for coronary calcification [29, 45]. In our study, we found higher serum levels of HIF-1a protein in SS/RS patients than in a healthy control group. Our results show similarities between the HIF-1a mRNA expression in monocytes and the HIF1 a protein levels in serum. The similarities suggest that monocytes may be partly responsible for the distribution of the HIF-1 a protein in serum and may therefore be potential targets for future therapeutic approaches.

In this study, we also found that HMOX-1 mRNA expression in monocytes was significantly increased in SS/RS patients compared to healthy control subjects. This might reflect a close interaction between HIF-1 and HMOX-1. HMOX-1 is a microsomal membrane enzyme that initiates the catabolism of free heme, thereby initiating the clearance of toxic ROS catalyzed by heme. Studies have shown increased HMOX-1 activity in hypoxic cells compared to normoxic cells [46]. HIF-1 mediates the transcriptional activation of the HMOX-1 gene in response to hypoxia [4]. HMOX-1 is believed to be a stress defense molecule. Several studies have demonstrated elevated levels of HMOX-1 mRNA expression in the fibroblasts of SS patients, which increases their reactivity to hypoxia [47]. Similar patterns seem to exist in the monocytes of SS/RS patients, suggesting a more sensitive and pronounced reaction to hypoxia than in healthy individuals. In several studies, HMOX-1 has been shown not only to act as a downstream effector enzyme, translating HIF-1's cytoprotective effect into action [48], but also to promote the expression of HIF-1 itself [6]. In the current study, HMOX-1 expression in monocytes expectedly followed similar patterns in both SS/RS treatment groups. This study provides evidence of increased HMOX-1 expression in patients treated with single-drug therapy after 24 weeks, as well as expression similar to that at the time of inclusion in the combination treatment group after 24 weeks. This provides further evidence of a close interaction between HIF-1 and HMOX-1 during the body's adaptation to hypoxia.

In this study, we also analyzed the monocyte expression of HIF2a mRNA, another isoform of the HIF family [48]. The aim was to further understand the role of HIF-2a in the hypoxia response. HIF2a mRNA expression in monocytes appeared to show the same expression patterns as HIF-1a in affected patients as well as the same patterns during the course of therapy [49], indicating a similar physiological role for both HIF-1 isoforms and suggesting that both isoforms are likely subject to similar regulatory mechanisms.

\section{LIMITATIONS}

The results of this study supported our hypothesis. However, our study has several limitations. First, patient recruitment in this study occurred throughout the duration of the year. Consequently, some patients were included in winter, when RS attacks are more commonly seen, while other patients where included during the warmer months. In addition, in future studies, it would be of interest to investigate the dynamics of HIF-1a expression throughout the course of treatment rather than at a single endpoint, especially as six months exceeds the known therapeutic effect of the prostaglandins used in single-drug therapy. Furthermore, while RS was clearly a distinctive feature of the included patients, the underlying SS varied considerably in its severity, suggesting that stricter inclusion criteria should be used in future studies. Moreover, there were significant differences in the gender composition and in the number of patients treated with immunomodulators and anticoagulants between the two therapy arms. Hence, our results may be subject to bias. Finally, patients at different stages of the disease were included. Through patient-wise pairing of the data and application of the respective paired statistical tests, this limitation was taken into account. However, given the statistically low number of patients, a confounding effect on the data cannot be ruled out. Finally, our data suggest that further investigation into clinical endpoints, such as patient outcome and the effects of the different therapy regimes on the severity and number of Raynaud attacks, would be valuable. This is the subject of an ongoing investigation.

\section{CONCLUSION}

We describe, for the first time (to our knowledge), significantly increased levels of HIF-1a mRNA expression in the monocytes, as well as elevated HIF-1a protein levels in the serum, of patients suffering from SS and RS compared to healthy control subjects. In a unique approach, we analyzed HIF-1a expression in the context of two different therapy regimes and provide evidence of a therapy-related difference in expression and serum levels. We also demonstrate a correlation between increased HIF-1a mRNA expression in monocytes and increased levels of an HIF-1 target, HMOX-1, indicating a close interaction between these factors and stressing the importance of HIF-1a mRNA expression in monocytes for the response to hypoxia.

In summary, HIF-1a shows promise as a potential indicator for the identification of SS/RS patients and possibly exhibits value as a marker for therapeutic efficacy. Our data warrant further validation in larger clinical trials.

\section{ACKNOWLEDGEMENTS}

This work was supported in part by the German Heart Foundation (project number K/ 26/13).

\section{AUTHOR CONTRIBUTIONS}

$\mathrm{LAH}$ and IA designed the study; $\mathrm{LAH}, \mathrm{MK}, \mathrm{MM}$ and DS performed the research; $\mathrm{LAH}$, $\mathrm{MK}, \mathrm{MH}, \mathrm{SR}, \mathrm{DD}, \mathrm{CB}, \mathrm{CH}$, and $\mathrm{IH}$ analyzed the data; and $\mathrm{LAH}$ and $\mathrm{MH}$ wrote the manuscript.

\section{ADDITIONAL INFORMATION}

Competing interests: The authors declare no competing interests.

Publisher's note: Springer Nature remains neutral with regard to jurisdictional claims in published maps and institutional affiliations.

\section{REFERENCES}

1. Semenza GL. Hypoxia-inducible factor 1: master regulator of $\mathrm{O} 2$ homeostasis. Curr Opin Genet Dev. 1998;8:588-94.

2. Semenza GL, Agani F, Booth G, Forsythe J, lyer N, Jiang BH, et al. Structural and functional analysis of hypoxia-inducible factor 1. Kidney Int. 1997;51:553-5.

3. Wang GL, Jiang BH, Rue EA, Semenza GL. Hypoxia-inducible factor 1 is a basichelix-loop-helix-PAS heterodimer regulated by cellular $\mathrm{O} 2$ tension. Proc Natl Acad Sci USA. 1995;92:5510-4.

4. Lee PJ, Jiang BH, Chin BY, lyer NV, Alam J, Semenza GL, et al. Hypoxia-inducible factor- 1 mediates transcriptional activation of the heme oxygenase- 1 gene in response to hypoxia. J Biol Chem. 1997;272:5375-81.

5. Hu J, Discher DJ, Bishopric NH, Webster KA. Hypoxia regulates expression of the endothelin-1 gene through a proximal hypoxia-inducible factor-1 binding site on the antisense strand. Biochem Biophys Res Commun. 1998;245:894-9.

6. Miao RZ, Liu LQ, Chen L, Li Z, Li LP, Guo RL, et al. Activity of heme oxygenase-1 affects expression levels of hypoxia inducible factor-1 gene in vitro. Chin Med J. 2012;125:1310-5

7. Semenza GL. HIF-1: mediator of physiological and pathophysiological responses to hypoxia. J Appl Physiol. 1985;2000:1474-80.

8. Bardos Jl, Ashcroft M. Negative and positive regulation of HIF-1: a complex network. Biochim Biophys Acta. 2005;1755:107-20. 
9. Deng W, Feng X, Li X, Wang D, Sun L. Hypoxia-inducible factor 1 in autoimmune diseases. Cell Immunol. 2016;303:7-15.

10. van Hal TW, van Bon L, Radstake TR. A system out of breath: how hypoxia possibly contributes to the pathogenesis of systemic sclerosis. Int J Rheumatol. 2011;2011:824972.

11. Grader-Beck T, Wigley FM. Raynaud's phenomenon in mixed connective tissue disease. Rheum Dis Clin North Am. 2005;31:465-81.

12. Belch JJ. Raynaud's phenomenon: its relevance to scleroderma. Ann Rheum Dis. 1991;50(Suppl 4):839-45.

13. Kaufman MW, All AC. Raynaud's disease: patient education as a primary nursing intervention. J Vasc Nurs. 1996;14:34-9.

14. Herrick A, Muir L. Raynaud's phenomenon (secondary). BMJ Clin Evid. 2014;2014: pii: 1125.

15. Sunderkotter C, Riemekasten G. Pathophysiology and clinical consequences of Raynaud's phenomenon related to systemic sclerosis. Rheumatology. 2006;45 (Suppl 3):iii33-5.

16. LeRoy EC, Medsger TA Jr. Raynaud's phenomenon: a proposal for classification. Clin Exp Rheumatol. 1992;10:485-8.

17. Distler JH, Jungel A, Pileckyte M, Zwerina J, Michel BA, Gay RE, et al. Hypoxiainduced increase in the production of extracellular matrix proteins in systemic sclerosis. Arthritis Rheum. 2007;56:4203-15.

18. Wei J, Bhattacharyya S, Tourtellotte WG, Varga J. Fibrosis in systemic sclerosis: emerging concepts and implications for targeted therapy. Autoimmun Rev. 2011;10:267-75.

19. Korn JH, Mayes M, Matucci Cerinic M, Rainisio M, Pope J, Hachulla E, et al. Digital ulcers in systemic sclerosis: prevention by treatment with bosentan, an oral endothelin receptor antagonist. Arthritis Rheum. 2004;50:3985-93.

20. Mohrland JS, Porter JM, Smith EA, Belch J, Simms MH. A multiclinic, placebocontrolled, double-blind study of prostaglandin E1 in Raynaud's syndrome. Ann Rheum Dis. 1985;44:754-60.

21. Creutzig A, Lehmacher W, Elze M. Meta-analysis of randomised controlled prostaglandin E1 studies in peripheral arterial occlusive disease stages III and IV. Vasa. 2004;33:137-44.

22. Matucci-Cerinic M, Denton CP, Furst DE, Mayes MD, Hsu VM, Carpentier $P$, et al. Bosentan treatment of digital ulcers related to systemic sclerosis: results from the RAPIDS-2 randomised, double-blind, placebo-controlled trial. Ann Rheum Dis. 2011;70:32-8.

23. Jaipersad AS, Lip GY, Silverman S, Shantsila E. The role of monocytes in angiogenesis and atherosclerosis. J Am Coll Cardiol. 2014;63:1-11.

24. Elbarghati L, Murdoch C, Lewis CE. Effects of hypoxia on transcription factor expression in human monocytes and macrophages. Immunobiology. 2008;213:899-908.

25. Sambo P, Jannino L, Candela M, Salvi A, Donini M, Dusi S, et al. Monocytes of patients with systemic sclerosis (scleroderma) spontaneously release in vitro increased amounts of superoxide anion. J Invest Dermatol. 1999;112:78-84.

26. Christmann RB, Lafyatis R. The cytokine language of monocytes and macrophages in systemic sclerosis. Arthritis Res Ther. 2010;12:146.

27. Ziello JE, Jovin IS, Huang Y. Hypoxia-Inducible Factor (HIF)-1 regulatory pathway and its potential for therapeutic intervention in malignancy and ischemia. Yale $J$ Biol Med. 2007;80:51-60.

28. Frede S, Stockmann C, Freitag P, Fandrey J. Bacterial lipopolysaccharide induces HIF-1 activation in human monocytes via p44/42 MAPK and NF-kappaB. Biochem J. 2006;396:517-27.

29. Li G, Lu WH, Ai R, Yang JH, Chen F, Tang ZZ. The relationship between serum hypoxia-inducible factor 1 alpha and coronary artery calcification in asymptomatic type 2 diabetic patients. Cardiovasc Diabetol. 2014;13:52.

30. Hull TD, Agarwal A, George JF. The mononuclear phagocyte system in homeostasis and disease: a role for heme oxygenase-1. Antioxid Redox Signal. 2014;20:1770-88.
31. Wenzel $P$, Rossmann $H$, Muller $C$, Kossmann $S$, Oelze $M$, Schulz A, et al. Heme oxygenase-1 suppresses a pro-inflammatory phenotype in monocytes and determines endothelial function and arterial hypertension in mice and humans. Eur Heart J. 2015:36:3437-46.

32. Hettema ME, Zhang D, Bootsma H, Kallenberg CG. Bosentan therapy for patients with severe Raynaud's phenomenon in systemic sclerosis. Ann Rheum Dis. 2007;66:1398-9.

33. Marasini B, Massarotti M, Bottasso B, Coppola R, Del Papa N, Maglione W, et al. Comparison between iloprost and alprostadil in the treatment of Raynaud's phenomenon. Scand J Rheumatol. 2004;33:253-6.

34. Wipff J, Dieude P, Avouac J, Tiev K, Hachulla E, Granel B, et al. Association of hypoxia-inducible factor 1A (HIF1A) gene polymorphisms with systemic sclerosis in a French European Caucasian population. Scand J Rheumatol. 2009;38:291-4

35. Higgins DF, Kimura K, Bernhardt WM, Shrimanker N, Akai Y, Hohenstein B, et al. Hypoxia promotes fibrogenesis in vivo via HIF-1 stimulation of epithelial-tomesenchymal transition. J Clin Invest. 2007;117:3810-20.

36. Wenger RH, Kvietikova I, Rolfs A, Gassmann M, Marti HH. Hypoxia-inducible factor-1 alpha is regulated at the post-mRNA level. Kidney Int. 1997;51:560-3.

37. Grygiel-Gorniak B, Puszczewicz M. Oxidative damage and antioxidative therapy in systemic sclerosis. Mediat Inflamm. 2014;2014:389582.

38. Pialoux V, Mounier R, Brown AD, Steinback CD, Rawling JM, Poulin MJ. Relationship between oxidative stress and HIF-1 alpha mRNA during sustained hypoxia in humans. Free Radic Biol Med. 2009;46:321-6.

39. Zhang Y, Li L, Hua Y, Nunn JM, Dong F, Yanagisawa M, et al. Cardiac-specific knockout of ET(A) receptor mitigates low ambient temperature-induced cardiac hypertrophy and contractile dysfunction. J Mol Cell Biol. 2012;4:97-107.

40. Niecknig H, Tug S, Reyes BD, Kirsch M, Fandrey J, Berchner-Pfannschmidt U. Role of reactive oxygen species in the regulation of HIF-1 by prolyl hydroxylase 2 under mild hypoxia. Free Radic Res. 2012;46:705-17.

41. Qutub AA, Popel AS. Reactive oxygen species regulate hypoxia-inducible factor 1alpha differentially in cancer and ischemia. Mol Cell Biol. 2008;28:5106-19.

42. Bellando-Randone S, Lepri G, Bruni C, Blagojevic J, Radicati A, Cometi L, et al. Combination therapy with Bosentan and Sildenafil improves Raynaud's phenomenon and fosters the recovery of microvascular involvement in systemic sclerosis. Clin Rheumatol. 2016:35:127-32.

43. Hafner F, Thomas G, Froehlich H, Steidl K, Brodmann M. Effect of a sequential therapy of bosentan and iloprost versus a monotherapy with bosentan in the treatment of scleroderma related digital ulcers. Int Angiol. 2011;30:493-5.

44. Cawello W, Leonhardt A, Schweer H, Seyberth HW, Bonn R, Lomeli AL. Dose proportional pharmacokinetics of alprostadil (prostaglandin E1) in healthy volunteers following intravenous infusion. $\mathrm{Br} \mathrm{J}$ Clin Pharmacol. 1995;40:273-6.

45. Xue L, Chen H, Lu K, Huang J, Duan H, Zhao Y. Clinical significance of changes in serum neuroglobin and HIF-1alpha concentrations during the early-phase of acute ischemic stroke. J Neurol Sci. 2017;375:52-7.

46. Ishii $\mathrm{T}$, Itoh $\mathrm{K}$, Takahashi S, Sato $\mathrm{H}$, Yanagawa $\mathrm{T}$, Katoh $\mathrm{Y}$, et al. Transcription factor Nrf2 coordinately regulates a group of oxidative stress-inducible genes in macrophages. J Biol Chem. 2000;275:16023-9.

47. Panchenko MV, Farber HW, Korn JH. Induction of heme oxygenase-1 by hypoxia and free radicals in human dermal fibroblasts. Am J Physiol Cell Physiol. 2000;278: C92-101.

48. Pachori AS, Melo LG, Zhang L, Solomon SD, Dzau VJ. Chronic recurrent myocardial ischemic injury is significantly attenuated by pre-emptive adenoassociated virus heme oxygenase- 1 gene delivery. J Am Coll Cardiol. 2006:47:635-43.

49. Loboda A, Jozkowicz A, Dulak J. HIF-1 and HIF-2 transcription factors--similar but not identical. Mol Cells. 2010;29:435-42. 